Research Article

\title{
Correlations of mRNA Levels among Efflux Transporters, Transcriptional Regulators, and Scaffold Proteins in Non-Small-Cell Lung Cancer
}

\author{
Xieyi Zhang $\mathbb{D}^{\mathbb{D}}{ }^{1}$ Wangyang Liu, ${ }^{2}$ Kazue Edaki, ${ }^{1}$ Yuta Nakazawa, ${ }^{1}$ Hiroki Kamioka, ${ }^{2}$ \\ Atsushi Fujita, ${ }^{3}$ Ryoichi Onozato, ${ }^{3}$ Misa Iijima, ${ }^{4}$ Shigeru Tsuchida, ${ }^{5}$ Takahiro Arai, ${ }^{6}$ \\ Yukiyoshi Fujita, ${ }^{6}$ Kenta Mizoi, ${ }^{1}$ and Takuo Ogihara ${ }^{1,2}$ \\ ${ }^{1}$ Laboratory of Biopharmaceutics, Department of Pharmacology, Faculty of Pharmacy, \\ Takasaki University of Health and Welfare, 60 Nakaorui-chou, Takasaki-shi, Gunma 370-0033, Japan \\ ${ }^{2}$ Laboratory of Clinical Pharmacokinetics, Graduate School of Pharmaceutical Sciences, \\ Takasaki University of Health and Welfare, 60 Nakaorui-chou, Takasaki-shi, Gunma 370-0033, Japan \\ ${ }^{3}$ Department of General Thoracic Surgery, Gunma Prefectural Cancer Center, 617-1 Takahayashinishi-chou, Ota-shi, \\ Gunma 373-0828, Japan \\ ${ }^{4}$ Department of Pathology and Clinical Laboratories, Gunma Prefectural Cancer Center, 617-1 Takahayashinishi-chou, Ota-shi, \\ Gunma 373-0828, Japan \\ ${ }^{5}$ Division of Clinical Laboratory, Gunma Prefectural Cancer Center, 617-1 Takahayashinishi-chou, Ota-shi, \\ Gunma 373-0828, Japan \\ ${ }^{6}$ Division of Pharmacy, Gunma Prefectural Cancer Center, 617-1 Takahayashinishi-chou, Ota-shi, Gunma 373-0828, Japan
}

Correspondence should be addressed to Xieyi Zhang; x-zhang@takasaki-u.ac.jp

Received 15 July 2021; Revised 8 November 2021; Accepted 13 November 2021; Published 28 November 2021

Academic Editor: Mohd Adnan

Copyright (c) 2021 Xieyi Zhang et al. This is an open access article distributed under the Creative Commons Attribution License, which permits unrestricted use, distribution, and reproduction in any medium, provided the original work is properly cited.

Multidrug resistance (MDR) due to enhanced drug efflux activity of tumor cells can severely impact the efficacy of antitumor therapies. We recently showed that increased activity of the efflux transporter P-glycoprotein (P-gp) associated with activation of Snail transcriptional regulators may be mediated mainly by moesin in lung cancer cells. Here, we aimed to systematically evaluate the relationships among mRNA expression levels of efflux transporters (P-gp, breast cancer resistance protein (BCRP), and multidrug resistance-associated protein 2 (MRP2)), scaffold proteins (ezrin (Ezr), radixin (Rdx), and moesin (Msn); ERM proteins), and SNAI family members (Snail, Slug, and Smac) in clinical lung cancer and noncancer samples. We found high correlations between relative (cancer/noncancer) mRNA expression levels of Snail and Msn, Msn and P-gp, Slug and MRP2, and Smuc and BCRP. These findings support our previous conclusion that Snail regulates P-gp activity via Msn and further suggest that Slug and Smuc may contribute to the functional regulation of MRP2 and BCRP, respectively, in lung cancer cells. This trial is registered with UMIN000023923.

\section{Introduction}

Lung cancer is the leading cause of cancer death in the United States. Indeed, almost one-quarter of all cancer deaths are due to lung cancer [1], and among patients with non-small-cell lung cancer (NSCLC), only $26 \%$ are alive for more than 5 years after diagnosis [2]. In many patients, surgical operation is not feasible, and thus, chemotherapy and adjuvant therapy are used as first-line treatments [3]. However, these treatments are often adversely affected by the emergence of multidrug resistance (MDR), i.e., resistance to multiple mechanistically and structurally unrelated antitumor drugs [4], resulting in a poor prognosis [5].

A major cause of MDR is increased activity of drug efflux transporters, such as P-glycoprotein (P-gp, ATP-binding cassette subfamily $\mathrm{B}$ member (ABCB1)), breast cancer 
resistance protein (BCRP, ATP-binding cassette subfamily G member 2 (ABCG2)), and multidrug resistance-associated protein 2 (MRP2, ATP-binding cassette subfamily C member 2 (ABCC2)), leading to reduced accumulation of anticancer drugs in cells [6]. P-gp was first identified as being associated with MDR in leukemia patients [7]. However, $\mathrm{P}$-gp is also present in normal tissues, where it has a protective role, mediating the efflux transport of xenobiotics. $\mathrm{P}$-gp functions as an efflux pump for a wide range of amphiphilic, bulky type II cationic drugs and other hydrophobic compounds, including endogenous and exogenous metabolites or toxins, steroid hormones, hydrophobic peptides, and even glycolipids [8]. BCRP has been suggested to be closely associated with resistance of NSCLC to topoisomerase I inhibitors, including irinotecan and its active metabolite, SN-38 [9], while MRP2 contributes to cisplatin resistance [10]. Recently, scaffold proteins, including ezrin (Ezr), radixin (Rdx), and moesin (Msn) (ERM proteins), have been shown to regulate the location of efflux transporters at the cell membrane, consequently regulating their functional activity [6]. They appear to serve as couriers to deliver the transporter molecules to the cell membrane [11-13]. In HCC827 human lung adenocarcinoma cells, Ezr or Msn knockdown significantly reduced Rhodamine123 (Rho123) efflux, which suggests that Ezr and/or Msn are involved in the regulation of P-gp activity in the lung [14].

Members of the SNAI family, including Snail (SNAI1), Slug (SNAI2), and Smuc (SNAI3), are considered as indicators of the malignancy of cancer [15]. Increased malignancy with loss of cell-cell adhesion is associated with epithelial-mesenchymal transition (EMT), which is the first step in the conversion of primary epithelial cells into mesenchymal cells [16]. EMT is associated with altered expression of various genes [17], and SNAI family members are transcriptional regulators that trigger EMT [18]. A positive correlation between Snail and BCRP has been found in breast cancer [19]. However, a recent study indicated that BCRP function was decreased via a reduction of the expression level in HCC827 lung cancer cells with Snail-induced EMT [20]. We previously found that Snailinduced EMT increased the membrane localization and activity of MRP5, but not those of MRP2, in HCC827 cells, resulting in increased cisplatin resistance [20]. We also showed that a histone deacetylase inhibitor, entinostat, suppresses not only Snail-induced cancer malignant alteration, but also P-gp-mediated MDR [21]. Moreover, Snail induces ERM proteins [14]. Transfection of lung cancer cells with Snail reduced the expression levels of epithelial markers, whereas expression of mesenchymal markers was increased [22]. Furthermore, the mRNA expression level of Msn, but not Ezr or Rdx, was increased [12]. Overexpression of Snail induced an increase in P-gp activity in HCC827 cells, and the increase in P-gp activity was suppressed by knockdown of Msn [12]. Thus, Snail may regulate Msn expression and thereby regulate the efflux activity of P-gp in the lung cancer HCC827 cell line [12, 22]. Moreover, these two studies confirmed that the mRNA and protein expression levels of $\mathrm{P}$-gp were consistent in both Mock- and Snail-overexpressing cells.
Although the relationships among transcription regulators, efflux transporters, and ERM proteins at the cellular level have been investigated to some extent, the clinically relevant associations are unknown [6]. Therefore, we set out to systematically evaluate the relationships among efflux transporters (P-gp, BCRP, and MRP2), ERM proteins, and SNAI family members in clinical samples of cancer and noncancer lung tissues from the same subjects in order to identify cancer-related changes. In the present work, we focused on mRNA expression levels in order to identify efflux transporter-regulatory mechanisms operating at the transcriptional level.

\section{Materials and Methods}

2.1. Clinical Samples. Surgically excised lung tissues, including cancer tissues and adjacent noncancer tissues, were obtained from 9 patients between 2016 and 2018 in the Gunma Prefectural Cancer Center (Gunma, Japan). Informed consent was obtained from each patient, and studies were performed with the approval of the Ethics Committee of Takasaki University of Health and Welfare (approval no. \#2906) and Gunma Prefectural Cancer Center (approval no. \#405-29042), in accordance with the Declaration of Helsinki. The study was preregistered in the University Hospital Medical Information Network (UMIN) clinical trial system (UMIN000023923). The characteristics of the nine patients included in the current study are summarized in Table 1 . The histopathological subtype was adenocarcinoma (AC) in five patients and squamous-cell carcinoma in four patients.

2.2. mRNA Extraction and cDNA Synthesis. mRNA was extracted from NSCLC cell lines seeded on 24-well culture plates using the reported method [23]. Lung tissue (about $200 \mathrm{mg}$ ) from patients was initially homogenized using a disposable homogenizer (Nippi, Tokyo, Japan). Total RNA was extracted using a NucleoSpin RNA Midi (MacheryNagel, Düren, Germany) according to the manufacturer's instructions. cDNA was synthesized from $1 \mathrm{mg}$ total RNA with ReverTraAce (Toyobo, Osaka, Japan) according to the manufacturer's instructions, using a T100 ${ }^{\mathrm{TM}}$ Thermal Cycler.

2.3. Quantitative Real-Time Polymerase Chain Reaction ( $q R T$ PCR). Quantitation of mRNA expression level by qRT-PCR was performed with Power SYBER ${ }^{\mathrm{TM}}$ Green PCR Master Mix (Applied Biosystems, CA, USA) using the MX3000P $\mathrm{P}^{\mathrm{TM}}$ Multiplex Quantitative PCR System (Stratagene, CA, USA). The mRNA expression levels of target genes were quantified relative to that of the housekeeping gene GAPDH according to the following equation (Ct: threshold line):

gene expression level $=1000 \times 0.5^{-(C t(\text { GAPDH })-C t(\text { target gene }))}$.

The absolute value was defined as the gene expression level in the cancer tissue. The relative value was defined as the ratio of the gene expression level in the cancer tissue to that in the noncancer tissue. 
TABLE 1: Characteristics of patients from whom samples were obtained.

\begin{tabular}{lccccc}
\hline Patient no. & Gender & Age (years) & Histological subtype & Stage & TNM classification \\
\hline 1 & Female & 63 & AC & IA & T1aN0M0 \\
2 & Male & 72 & AC & IA & T1bN0M0 \\
3 & Male & 75 & SCC & IIA & T2aN1M0 \\
4 & Female & 71 & AC & IIIA & T1bN0M0 \\
5 & Male & 63 & SCC & IB & T2aN2M0 \\
6 & Male & 76 & ACC & IA2 & T1bN0M0 \\
7 & Female & 72 & AC & IB & T2aN0M0 \\
8 & Male & 68 & SCC & IB & T2aN0M0 \\
9 & Male & 76 & & & \\
\hline
\end{tabular}

AC: adenocarcinoma; SCC: squamous-cell carcinoma.

2.4. Statistical Analysis. All results are presented as mean\pm standard deviation (SD). Pearson's test or the Spearman correlation test was used to obtain correlation coefficients. The criterion of statistical significance was $p<0.05$.

\section{Results}

3.1. Correlations of $m R N A$ Expression Levels in Cancer Tissues (Absolute Values). Expression levels of Snail mRNA were highly correlated with $\mathrm{Rdx}(r=0.667)$ and Msn $(r=0.700)$ in lung cancer tissues. Slug mRNA levels showed a high correlation with MRP2 $(r=0.800)$, and Smuc mRNA levels showed a high correlation with BCRP $(r=0.818)$ (Figure 1$)$.

Expression levels of Rdx and Msn mRNAs showed very high correlations with P-gp ( $r=0.929$ for $\mathrm{Rdx}$ and $r=0.912$ for Msn). In addition, ERM protein expression levels were highly correlated with each other (Ezr vs. Rdx, $r=0.817$; Ezr vs. Msn, $r=0.783$; Rdx vs. Msn, $r=0.917)$.

3.2. Correlations of $m R N A$ Expression Levels in Cancer and Noncancer Tissues (Relative Values). The relative (cancer/ noncancer) mRNA expression values showed a high correlation between Snail and Msn $(r=0.792)$ and also between Msn and P-gp $(r=0.866)$. Slug and MRP2 showed an extremely high correlation $(r=0.949)$, and Smuc and BCRP were also highly correlated $(r=0.871)$ (Figure 2$)$. However, in contrast to the absolute values, significant correlations were not found between $\mathrm{Rdx}$ and Snail or between Rdx and P-gp. There was also no correlation among ERM proteins.

3.3. Correlations between Relative and Absolute Values of $m R N A$ Expression. The relative and absolute values were highly correlated for Snail $(r=0.679)$, Slug $(r=0.918)$, Msn $(r=0.990)$, P-gp $(r=0.905)$, and MRP2 $(r=0.958)$, but not for Smuc, Ezr, Rdx, or BCRP.

\section{Discussion}

mRNA expression level is often used as an indicator of protein expression and function [24]. In our previous study, we reported that Snail-overexpressing HCC827 cells showed significantly increased mRNA expression of Msn compared with Mock cells, whereas the expression levels of Ezr and $\mathrm{Rdx}$ were unchanged [12]. The protein expression level of Msn was consistent with the mRNA expression level.
Moreover, Msn knockdown blocks the increase in P-gp activity in HCC827 cells. Independently, we have also found that P-gp function is regulated by the ERM proteins. Our present findings are consistent with that report. We also confirmed a high correlation of relative values of mRNA expression levels between Snail with Msn in NSCLC.

We further investigated whether the mRNA expression levels of ERM proteins were correlated with that of P-gp. As regards relative values, only Msn was correlated with P-gp mRNA expression. However, we previously found that, in HCC827 cells, Ezr or Msn knockdown significantly reduced the Rho123 efflux rate, suggesting that Ezr and/or Msn are involved in the membrane expression of P-gp in the lung [14]. This is reasonable, since it is well known that mRNA expression levels of transporters do not necessarily correlate with protein expression level or functional activity [25]. Moreover, P-gp function in clinical samples may change depending on factors such as the age and disease status of the patients.

$\mathrm{Rdx}$ regulates MRP2 function in the liver, both in normal tissues and in cancer cells [26]. Reduced phosphorylation of threonine at the C-terminal of Rdx impairs the binding of MRP2 to actin, which decreases the localization of MRP2 to the cell membrane [27]. BCRP function was evaluated using NSCLC HCC827 cells and was found to be correlated with expression of Ezr and Msn [14]. Here, we found that the mRNA expression of MRP2 was positively correlated with that of Slug, and the mRNA expression of BCRP was positively correlated with that of Smuc, for both absolute and relative values. However, no relationship was observed between MRP2/BCRP and ERM proteins. Our results suggest that Slug and Smuc may contribute to the functional regulation of MRP2 and BCRP, respectively, in lung cancer patients.

There are several limitations in the present study. Firstly, we used only clinical samples, because it is difficult to acquire the lung tissue from normal subjects. Secondly, we measured mRNA expression levels of efflux transporters, which may be useful to identify regulatory mechanisms operating at the transcriptional level, although it should be noted that they are not necessarily reflected in changes of protein expression or functional activity. Further studies using lung cancer cell lines are underway to address these issues. Moreover, only nine patients, mostly with early-stage disease based on their TNM classifications, were included in this study. Further clinical samples, especially from patients with advanced metastatic tumors, are needed to confirm our findings. 


\begin{tabular}{|c|c|c|c|c|c|c|c|c|c|c|c|}
\hline & & Snail & Slug & Smuc & Ezrin & Radixin & Moesin & P-gp & MRP2 & BCRP & $\begin{array}{c}\text { Relative } \\
\text { value }\end{array}$ \\
\hline & $\mathrm{R} / \mathrm{A}$ & & -0.210 & -0.211 & -0.133 & -0.152 & $\underline{0.792}$ & 0.626 & -0.159 & -0.352 & Snail \\
\hline Snail & & $\underline{0.679}$ & & -0.210 & 0.130 & 0.136 & 0.208 & 0.000 & $\underline{0.949}$ & -0.261 & Slug \\
\hline Slug & -0.417 & & $\underline{0.918}$ & & -0.211 & 0.237 & -0.277 & -0.202 & 0.030 & $\underline{0.871}$ & Smuc \\
\hline Smuc & 0.059 & 0.167 & & 0.494 & & 0.854 & -0.017 & 0.336 & 0.030 & 0.099 & Ezrin \\
\hline Ezrin & 0.650 & -0.033 & -0.276 & & 0.036 & & 0.018 & 0.192 & 0.188 & 0.531 & Radixin \\
\hline Radixin & $\underline{0.667}$ & -0.033 & -0.176 & $\underline{0.817}$ & & 0.037 & & $\underline{0.866}$ & 0.239 & -0.315 & Moesin \\
\hline Moesin & $\underline{0.700}$ & 0.050 & -0.017 & $\underline{0.783}$ & $\underline{0.917}$ & & $\underline{0.990}$ & & -0.033 & -0.192 & P-gp \\
\hline P-gp & 0.485 & 0.159 & -0.172 & 0.644 & $\underline{0.929}$ & $\underline{0.912}$ & & $\underline{0.905}$ & & 0.695 & MRP2 \\
\hline MRP2 & 0.000 & $\underline{0.800}$ & 0.485 & 0.250 & 0.167 & 0.233 & 0.167 & & $\underline{0.958}$ & & BCRP \\
\hline BCRP & 0.131 & 0.429 & $\underline{0.818}$ & -0.238 & 0.048 & 0.214 & 0.144 & 0.643 & & 0.525 & \\
\hline $\begin{array}{l}\text { Absolute } \\
\text { value }\end{array}$ & Snail & Slug & Smuc & Ezrin & Radixin & Moesin & P-gp & MRP2 & BCRP & & \\
\hline
\end{tabular}

FIGURE 1: Correlations ( $r$ values) among absolute and relative mRNA expression levels of SNAI family members, ERM proteins, and efflux transporters in clinical lung cancer tissue samples. Each value in the part below the oblique line shows the correlation of the absolute values of the parameters in the corresponding row and column, and those in the part above the oblique line show the correlation of the relative values of the parameters in the corresponding row and column. The values on the oblique line show the correlation between relative and absolute values (R/A) of the same parameter. The boldface underline indicates $p<0.05$.
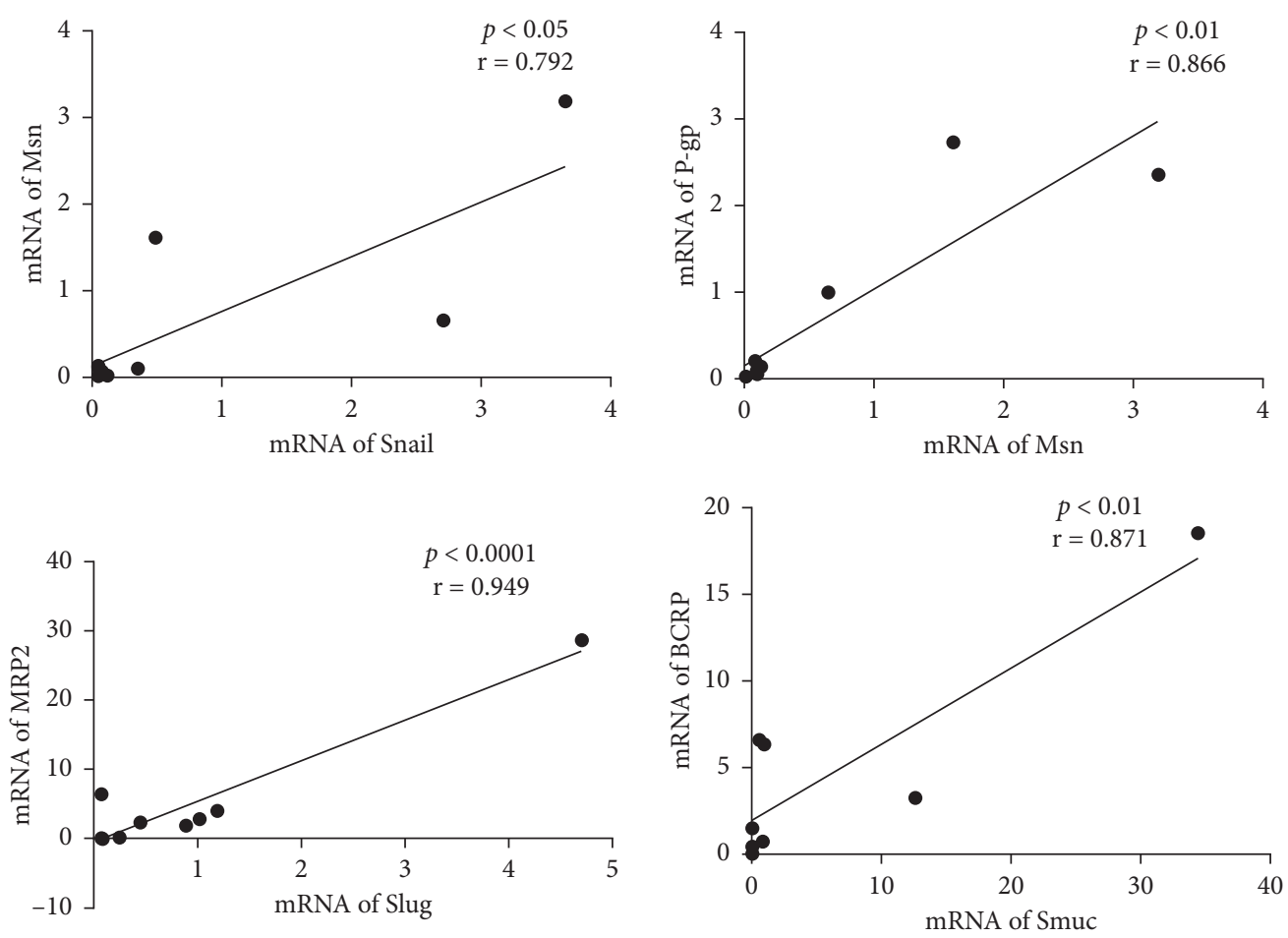

Figure 2: Correlations of relative mRNA expression levels of Snail and Msn, Msn and P-gp, Slug and MRP2, and Smuc and BCRP. 


\section{Conclusions}

In conclusion, high correlations were found between the relative mRNA expression levels of Snail and Msn and Msn and P-gp, suggesting that the Snail/Msn pathway contributes to the functional regulation of P-gp. Our findings also suggest that Slug and Smuc may contribute to the functional regulation of MRP2 and $\mathrm{BCRP}$, respectively, in lung cancer tissues.

\section{Data Availability}

The data used to support the findings of this study are included within the article.

\section{Conflicts of Interest}

The authors declare they have no conflicts of interest.

\section{Acknowledgments}

The authors thank Mr. Kentaro Yano for his support. Thanks are due to the members of the Department of Pharmaceutical Science of Takasaki University of Health and Welfare for data analysis and advice on the manuscript. This work was supported by the JSPS KAKENHI (Grant no. 20K22717) and the Ichiro Kanehara Foundation for the Promotion of Medical Sciences and Medical Care.

\section{References}

[1] R. L. Siegel, K. D. Miller, H. E. Fuchs, and A. Jemal, "Cancer statistics, 2021," CA: A Cancer Journal for Clinicians, vol. 71, no. 1, pp. 7-33, 2021.

[2] D. S. Ettinger, D. E. Wood, and D. L. Aisner, "NCCN guidelines insights: non-small cell lung cancer, version 2.2021: featured updates to the NCCN guidelines," Journal of the National Comprehensive Cancer Network, vol. 19, no. 3, pp. 254-266, 2021.

[3] D. Rocco, L. Della Gravara, C. Battiloro, and C. Gridelli, "The role of nivolumab combined to immunotherapy and/or chemotherapy in the first-line treatment of advanced Non Small Cell Lung Cancer," Expert Opinion on Biological Therapy, vol. 21, no. 3, pp. 303-309, 2021.

[4] Y.-K. Zhang, Y.-J. Wang, P. Gupta, and Z.-S. Chen, "Multidrug resistance proteins (MRPs) and cancer therapy," The AAPS Journal, vol. 17, no. 4, pp. 802-812, 2015.

[5] J. Wan, X. a. Ling, J. Wang, G. g. Ding, and X. Wang, "Inhibitory effect of Ubenimex combined with fluorouracil on multiple drug resistance and P-glycoprotein expression level in non-small lung cancer," Journal of Cellular and Molecular Medicine, vol. 24, no. 21, pp. 12840-12847, 2020.

[6] T. Ogihara, K. Mizoi, H. Kamioka, and K. Yano, "Physiological roles of ERM proteins and transcriptional regulators in supporting membrane expression of efflux transporters as factors of drug resistance in cancer," Cancers, vol. 12, 2020.

[7] J. Holmes and R. West, "The effect of MDR-1 gene expression on outcome in acute myeloblastic leukaemia," British Journal of Cancer, vol. 69, no. 2, pp. 382-384, 1994.

[8] A. D. Mottino and V. A. Catania, "Hepatic drug transporters and nuclear receptors: regulation by therapeutic agents,"
World Journal of Gastroenterology, vol. 14, no. 46, pp. 7068-7074, 2008.

[9] Y. Fan, N. Mansoor, T. Ahmad et al., "Enzyme and transporter kinetics for CPT-11 (irinotecan) and SN-38: an insight on tumor tissue compartment pharmacokinetics using PBPK," Recent Patents on Anti-cancer Drug Discovery, vol. 14, no. 2, pp. 177-186, 2019.

[10] V. Materna, P. S. Holm, M. Dietel, and H. Lage, "Kinetic characterization of ribozymes directed against the cisplatin resistance-associated ABC transporter cMOAT/MRP2/ ABCC2," Cancer Gene Therapy, vol. 8, no. 3, pp. 176-184, 2001.

[11] H. Hayashi, A. Tamura, D. Krishnan et al., "Ezrin is required for the functional regulation of the epithelial sodium proton exchanger, NHE3," PLoS One, vol. 8, no. 2, Article ID e55623, 2013.

[12] H. Kamioka, T. Tomono, A. Fujita et al., "Moesin-mediated P-glycoprotein activation during snail-induced epithelialmesenchymal transition in lung cancer cells," Journal of Pharmaceutical Sciences, vol. 109, no. 7, pp. 2302-2308, 2020.

[13] T. Nakano, S. Sekine, K. Ito, and T. Horie, "Ezrin regulates the expression of Mrp2/Abcc2 and Mdr1/Abcb1 along the rat small intestinal tract," American Journal of Physiology-Gastrointestinal and Liver Physiology, vol. 305, no. 11, pp. G807-G817, 2013.

[14] K. Yano, C. Okabe, K. Fujii, Y. Kato, and T. Ogihara, "Regulation of breast cancer resistance protein and P-glycoprotein by ezrin, radixin and moesin in lung, intestinal and renal cancer cell lines," Journal of Pharmacy and Pharmacology, vol. 72, no. 4, pp. 575-582, 2020.

[15] A. Jayachandran, M. Konigshoff, H. Yu et al., "SNAI transcription factors mediate epithelial-mesenchymal transition in lung fibrosis," Thorax, vol. 64, no. 12, pp. 1053-1061, 2009.

[16] E. S. Cho, H. E. Kang, N. H. Kim, and J. I. Yook, "Therapeutic implications of cancer epithelial-mesenchymal transition (EMT)," Archives of Pharmacal Research, vol. 42, no. 1, pp. 14-24, 2019.

[17] R. Xu, J. Y. Won, C. H. Kim, D. E. Kim, and H. Yim, "Roles of the phosphorylation of transcriptional factors in epithelialmesenchymal transition," Journal of oncology, vol. 2019, Article ID 5810465, 2019.

[18] B. Gras, L. Jacqueroud, A. Wierinckx et al., "Snail family members unequally trigger EMT and thereby differ in their ability to promote the neoplastic transformation of mammary epithelial cells," PLoS One, vol. 9, no. 3, Article ID e92254, 2014.

[19] W.-J. Chen, H. Wang, Y. Tang, C.-L. Liu, H.-L. Li, and W.-T. Li, "Multidrug resistance in breast cancer cells during epithelial-mesenchymal transition is modulated by breast cancer resistant protein," Chinese Journal of Cancer, vol. 29, no. 2, pp. 151-157, 2010.

[20] K. Yano, I. Todokoro, H. Kamioka, T. Tomono, and T. Ogihara, "Functional alterations of multidrug resistanceassociated proteins 2 and 5, and breast cancer resistance protein upon snail-induced epithelial-mesenchymal transition in HCC827 cells," Biological and Pharmaceutical Bulletin, vol. 44, no. 1, pp. 103-111, 2021.

[21] T. Tomono, T. Machida, H. Kamioka, Y. Shibasaki, K. Yano, and T. Ogihara, "Entinostat reverses P-glycoprotein activation in snail-overexpressing adenocarcinoma HCC827 cells," PLoS One, vol. 13, no. 7, Article ID e0200015, 2018.

[22] T. Tomono, K. Yano, and T. Ogihara, "Snail-induced epithelial-to-mesenchymal transition enhances P-gp-Mediated multidrug resistance in HCC827 cells," Journal of Pharmaceutical Sciences, vol. 106, no. 9, pp. 2642-2649, 2017. 
[23] T. Kobori, S. Fujiwara, K. Miyagi et al., "Involvement of moesin in the development of morphine analgesic tolerance through P-glycoprotein at the blood-brain barrier," Drug Metabolism and Pharmacokinetics, vol. 29, no. 6, pp. 482-489, 2014.

[24] M. Schena, D. Shalon, R. W. Davis, and P. O. Brown, "Quantitative monitoring of gene expression patterns with a complementary DNA microarray," Science, vol. 270, no. 5235, pp. 467-470, 1995.

[25] A. L. Bauernfeind and C. C. Babbitt, "The predictive nature of transcript expression levels on protein expression in adult human brain," BMC Genomics, vol. 18, no. 1, p. 322, 2017.

[26] S. Kikuchi, M. Hata, K. Fukumoto et al., "Radixin deficiency causes conjugated hyperbilirubinemia with loss of Mrp2 from bile canalicular membranes," Nature Genetics, vol. 31, no. 3, pp. 320-325, 2002.

[27] J. Saeki, S. Sekine, and T. Horie, "LPS-induced dissociation of multidrug resistance-associated protein 2 (Mrp2) and radixin is associated with Mrp2 selective internalization in rats," Biochemical Pharmacology, vol. 81, no. 1, pp. 178-184, 2011. 